\title{
Prospective associations of depression with survival: a population-based cohort study in patients with newly diagnosed breast cancer
}

\author{
Andrea Vodermaier • Wolfgang Linden • \\ Katerina Rnic $\cdot$ Sandra N. Young $\cdot$ Alvina Ng • \\ Nina Ditsch $\cdot$ Robert Olson
}

Received: 26 September 2013/ Accepted: 25 November 2013/Published online: 13 December 2013

(C) The Author(s) 2013. This article is published with open access at Springerlink.com

\begin{abstract}
Psychological factors may influence survival in breast cancer patients but results of previous research are inconclusive. This prospective population-based study tested whether depression predicts mortality in breast cancer patients. Routinely collected depression screening data were merged with electronically archived provincial cancer registry data and censored data from British Columbia Vital Statistics (extracted in December 2012). Cox proportional-hazards regression analyses were conducted to predict all-cause and breast cancer-specific mortality as a function of depression after controlling for biomedical confounders. Of 1,646 patients, 1,604 had breast cancer stages I-III and 42 had stage IV breast cancer. $176(11.0 \%)$ versus $28(66.7 \%)$ were deceased after a median follow-up of 76 months. In patients with curable breast cancer, depression predicted all-cause $(\mathrm{HR}=1.54$ (95\% CI 1.06-2.25); $p=0.024$ ), but not breast cancer-
\end{abstract}

A. Vodermaier $(\bowtie) \cdot$ W. Linden $\cdot$ K. Rnic

S. N. Young · A. Ng

Department of Psychology, University of British Columbia,

2136 West Mall, Vancouver, BC V6T 1Z4, Canada

e-mail: avoderma@psych.ubc.ca

A. Vodermaier $\cdot$ N. Ditsch

Department of Obstetrics and Gynecology-Campus

Grosshadern, University of Munich, Munich, Germany

W. Linden

BC Cancer Agency, Vancouver, BC, Canada

\section{S. N. Young}

Department of Psychology, University of Calgary, Calgary, AB, Canada

R. Olson

Centre for the North, BC Cancer Agency, Prince George, BC, Canada specific mortality $(\mathrm{HR}=1.51 \quad(95 \%$ CI $0.95-2.41)$; $p=0.084)$. No association was shown for metastatic disease. Stage-specific analyses demonstrated a 2-2.5-fold increase in breast cancer-specific and all-cause mortality in patients with stage I and II disease, but not in patients with stage III or IV breast cancer. In stage I breast cancer patients, age moderated effects of depression such that depressed younger patients diagnosed at age 45 (i.e., mean age $-1 \mathrm{SD})$ showed a ninefold $(\mathrm{HR}=9.82(95 \% \mathrm{CI}$ $2.26-42.68) ; p=0.002)$ increase in all-cause mortality and depressed patients at 57 a 3.7-fold (HR $=3.69(95 \%$ CI $1.44-9.48) ; p=0.007$ ) increase, while no association was evident in older patients at age 69 (mean age $+1 \mathrm{SD}$ ). Depression is strongly associated with mortality in younger patients with early stage breast cancer.

Keywords Breast cancer - Survival - Depression · Mortality $\cdot$ Age

\section{Introduction}

The question of whether psychological factors increase the likelihood of developing cancer and can influence the course of the disease has been of interest for the past three decades [1]. Meta-analytic data demonstrates that depression is a chronic problem in cancer patients and differentiates them from the general population [2]. Because cancer patients are more likely to present with ongoing depressive symptoms, depression may have a greater propensity to predict their mortality [3]. Recent meta-analyses of prospective research with mixed types of cancers indicate that depression at the time of diagnosis is associated with an increase in mortality $[4,5]$. However, the majority of past findings are difficult to compare because of a lack of 
control for biomedical prognosticators, use of mixed patient samples with various tumor types, and differences in the time point of depression assessment. Therefore, the present study tests associations of depression at the time of diagnosis with mortality in a large population-based sample of women with breast cancer.

Although the question of whether or not psychological factors predict mortality in women with breast cancer has been widely studied, findings are not consistent. Most studies based on patient self-report of depression using questionnaires failed to identify a relationship between depression and survival [6-11], whereas two studies demonstrated prospective associations of depression with survival $[12,13]$. Interestingly, studies that used depression scales validated for patients with cancer [14] such as the Hospital Anxiety and Depression Scale [15] or the Center for Epidemiological Studies Depression Scale [16] were more likely to identify a relationship of depression and mortality [12, 13]. Based on clinician diagnoses, large retrospective epidemiological studies also reported a significant relationship between depression and survival in breast cancer patients, such that depression was associated with a 1.3-1.4-fold decrease in survival $[17,18]$.

Furthermore, the time point of depression assessment also may contribute to random error in findings. Most cross-sectional studies where depression was measured at various time points after diagnosis failed to identify an association [10, 11], likely due to differences in where patients were in the cancer care trajectory, which likely influences concomitant mood symptoms. As well, the recency of follow-up merits attention in interpreting inconclusive findings among studies. A study that investigated depression as a predictor of mortality at longer follow-ups, that is, 5 and 10 years after diagnosis, demonstrated a significant association at 5 years, but not at 10 years, after diagnosis $[9,12]$. The decrease in strength of an association over several years may be rooted in the sensitivity to change of depression ratings, such that a single rating loses predictive validity over time. Finally, high survival rates of breast cancer and a minority of patients presenting clinical symptoms of depression create problems for more recent studies in attaining high statistical power and reliability.

Interestingly, the interaction of depression with age in predicting survival has rarely been studied, despite evidence that the risk of local recurrences, distant metastases and survival depend on the patients' age at diagnosis [1921]. Since prognoses for younger women tend to be worse [19-21], the disease is more likely perceived as threatening and therefore potentially more likely to result in depression and to be accompanied by a stronger biologic stress response in these women.
Additional factors likely play a role in breast cancer survivorship of younger women, since differences in breast cancer survival rates are only in part due to a greater likelihood of younger women being diagnosed with a more aggressive cancer that is usually at a later stage [21]. For example, the diagnosis of breast cancer may not have as severe an impact on the functional status or life expectancy of older as compared to younger women [19-21]. Coupled with the fact that depression often diminishes with age [22], this suggests that a diagnosis of breast cancer might be less likely to activate a stress response in older women. In contrast, a cancer diagnosis represents a drastic change in life expectancy [19-21] and quality of life for younger women and may be their first serious illness. Breast cancer treatments differ according to patients' age as well. On average, younger women with breast cancer are more likely to be treated with chemotherapy as well as with more radical hormonal treatment such as GnRH analogs. These create more severe side effects than a tamoxifen or aromatase inhibitor-only therapy, which are routinely prescribed to postmenopausal women with breast cancer. These side effects are likely accompanied by greater psychological consequences and poor adherence, which may further contribute to increased mortality. As a consequence of these myriad factors, depression may have greater predictive utility for mortality in younger cancer patients [23].

\section{The present study}

The purpose of this prospective cohort study was to test associations between depression (assessed after diagnosis but before treatment initiation) with breast cancer-specific and overall survival in women with newly diagnosed breast cancer. This design ensures that the psychological assessment is not confounded with emotional changes arising from the treatment itself; instead it mirrors the patients' personal resources at the time of cancer diagnosis, represents a marker of pre-morbid psychological functioning and reflects individual differences in stress response. We hypothesized that (1) depression predicts breast cancerspecific and all-cause mortality; (2) age moderates the relationship between depression and mortality, with a younger age and the presence of symptoms of depression being associated with the greatest risk of mortality.

\section{Methods}

\section{Procedures}

Within a routine screening program for emotional distress instituted in 2004, study participants were recruited consecutively at two urban cancer centers between 2004 and 
2009. Patients were routinely assessed for emotional distress after cancer diagnosis but before treatment initiation on their first day in the cancer clinic's hospital, before they were given detailed prognostic information and a treatment plan. The recruitment process is described in more detail elsewhere [23, 24]. These psychosocial data were merged with electronically archived demographic and biomedical data, as well as censored data extracted in December 2012 regarding the patients' survival status. This study received ethics approval from the local ethics board.

Patients and eligibility

A total of 2,441 patients with breast cancer were initially entered in the study. Eleven men with breast cancer were excluded, because male breast cancer is a very unique condition and difficult to compare with female breast cancer. Another 564 patients were excluded from the analysis because they had noninvasive breast cancer (carcinoma in situ) resulting in 1,866 eligible patients with invasive female breast cancer stages I-IV. Because of incomplete data in the Psychosocial Screen for Cancer, another 220 individuals were excluded from analyses resulting in a final sample of 1,646. Responders $(\mathrm{M}=56.19, \mathrm{SD}=12.54)$ and non-responders $(\mathrm{M}=58.80, \mathrm{SD}=13.38)$ differed in age $(t(268.38)=2.72, p=0.007)$, such that the patients included were 2.6 years younger on average. Responders and non-responders did not differ with respect to their living situation (i.e., living alone versus not living alone), which can be considered a proxy for marital status. Nor were there any differences between responders and non-responders in terms of biomedical and treatment variables (all $p$ 's $>0.05$ ). Median follow-up was 76 months.

\section{Measures}

The 21-item Psychosocial Screen for Cancer (PSSCAN) $[27,28]$ assesses anxiety and depression symptoms based on DSM-IV-TR criteria [29], perceived social support, desired social support, and quality of life. The scale was specifically developed for use with cancer patients. The questionnaire's reliability and validity, including empirically derived cut-offs and norms, have been reported [25, 26] and meet a high standard of psychometric quality [14]. Only the depression subscale was of interest in the context of the present study, and anxiety, social support, and quality of life at diagnosis were not examined.

\section{Depression}

Subscale scores can range from 5 to 25, with higher scores indicating more severe levels of depression, and with cutoffs of 8 and 11 signifying subclinical and clinical thresholds of depression, respectively. In comparison with the Hospital Anxiety and Depression Scale [15], sensitivity and specificity for the detection of a clinical level of depression were $98 \%$ for sensitivity and $86 \%$ for specificity, respectively. Two-month test-retest-reliability was $r=0.61$ [25].

Previous validation research found that both cancer outpatients $(d=0.26, p<0.001)$ as well as cancer inpatients $(d=0.70, p<0.001)$ reported significantly higher levels of depression compared to a community sample [25]. Age was negatively associated with depression in patients with cancer $(\rho=-0.157, p<0.001)$ as well as in a healthy community sample $(\rho=-0.190, p<0.001)$ (unpublished data derived from the validation samples). The PSSCAN questionnaire was validated in a mixed sample of cancer outpatients and inpatients, which included breast cancer patients.

\section{Statistical analyses}

Survival data were obtained from the Provincial Vital Statistics Agency, which provides complete death lists for all deceased British Columbians on a monthly basis. The censor date, which is the most recent date that death information was known, contains complete information on all patients in the registry, and all cases are censored at that same date. Censored cases were defined by non-receipt of information about a patient's death and death date at the time of retrieval of survival data. Thus, patients on whom data on survival status had been missing were categorized as censored. In this process the censoring mechanism is not related to the outcome and the assumption of non-informative as well as right censoring is satisfied.

Survival time was calculated by subtracting the date of death or censoring from the date of diagnosis. Analyses were conducted for death resulting from any cause (i.e., allcause mortality) and death resulting from breast cancer (i.e., breast cancer-specific mortality).

Cox proportional-hazards regression analysis uses the likelihood-ratio statistic and calculates the overall Chi-square. Parameter estimates for each variable in the model represent hazard ratios. Cox proportional-hazards regression analyses were conducted controlling for age, stage, grade, histology, estrogen receptor, her2-neu status, and treatment variables (i.e., surgery, radiation, chemotherapy, hormonal therapy). Given the fact that estrogen and progesterone receptor status was correlated at $\rho=0.53$, creating problems of multicollinearity, analyses only controlled for estrogen receptor status since it is more closely related to survival than progesterone receptor status. Furthermore, since treatments for breast cancer have changed over time and patients of this study were recruited within a 4-year time frame, we initially included year of diagnosis as a covariate in the model. However, due to non-significance, this covariate was ultimately excluded. 
In a first step, proportionality of hazards was examined by testing the time-dependency of each control or predictor variable separately. The respective variable was entered together with its interaction with time in a Cox regression model. If the variable $\times$ time interaction turned out to be significant, this respective variable demonstrates timedependent changes in hazards, and a variable $\times$ time interaction therefore needs to be included in the multivariate model [27]. Significant time-dependent covariates were eventually removed if they did not turn out to be significant in the multivariate model.

Next, a forced entry hierarchical model was chosen where age was entered in a first step, biomedical (i.e., stage (if applicable), grade, histology, estrogen receptor, her2 status) entered second, treatment variables (surgery, radiation, chemotherapy, hormonal therapy) entered third, depression entered fourth, and a depression by age interaction entered in the last step.

Age, grade, estrogen receptor status, and her2-neu status were entered as continuous variables into the Cox regression equation. Age was mean centered. All other variables (i.e., histology, surgery, upfront radiation, initial chemotherapy, hormonal therapy, depression) were entered as categorical variables. Depression was categorized at the clinical threshold of 11 and above on the PSSCAN depression subscale. The depression by age interaction contained depression as a binary variable and age as a mean centered continuous variable. This analysis was conducted on the full sample of stage I-III patients, and was conducted as separate analyses by tumor stage.

To test the significant interaction of depression with age in patients with stage I breast cancer, the model was rerun with age centered one standard deviation below the mean (at age 45) and age centered one standard deviation above the mean (at age 69).

Significant main and interaction effects were visualized by plotting Kaplan-Meier curves. For this purpose continuous variables (i.e., age) were categorized one standard deviation below the mean and less (45 and younger), one standard deviation below to one standard deviation above the mean (from 46 to 68), and one standard deviation above the mean (69 and older). All statistical tests were two-tailed and a $p$ value of $<0.05$ was considered statistically significant. Confidence intervals are also reported as an alternative indicator of significance indicating that the population mean will fall within the respective range in $95 \%$ of samples. Statistical analyses were conducted with IBM SPSS Statistics Version 21.

\section{Results}

Patients

As shown in Table 1, patients' mean age was 56.19 $(\mathrm{SD}=12.54)$. 1,604 $(97.4 \%)$ had non-metastatic breast cancer (stages I-III) at the time of breast cancer diagnosis and $42(2.6 \%)$ were primary metastasized (stage IV). The largest group of patients (804 $(48.8 \%)$ ) had stage I breast cancer. $370(22.5 \%)$ patients lived on their own. 562 $(34.1 \%)$ presented with depressive symptoms, and 260 $(15.8 \%)$ of these patients met clinical levels of depression. As of December 2012, $204(12.4 \%)$ patients were deceased, of which 126 (7.7\%) had died of breast cancer. Median length of survival at the time of data extraction was 74 months. Median follow-up was 76 months.

Prediction of breast cancer-specific and all-cause mortality (stages I-III)

Joint analysis of patients with curable breast cancer (stages I-III) demonstrated a main effect of depression on allcause mortality but not on breast-cancer specific mortality, such that depression at the time of cancer diagnosis increased hazards of all-cause mortality by $50 \%$ compared to non-depressed patients $(\mathrm{HR}=1.54 \quad(95 \%$ CI $1.06-2.25), p=0.024$ ) while the effect on breast cancerspecific mortality demonstrated an effect of similar magnitude which, however, only reached marginal significance $(\mathrm{HR}=1.51(95 \%$ CI 0.95-2.41), $p=0.084)($ see Table 2 and Fig. 1). Age did not moderate associations between depression and breast cancer-specific or all-cause mortality in patients with breast cancer stages I-III.

\section{Separate analyses by tumor stage}

Separate analyses by tumor stage revealed that depression had an impact on breast cancer-specific mortality and allcause mortality in patients with stage I and stage II breast cancer, but not in patients with stage III or IV breast cancer. As displayed in Table 3, the hazards of breast cancer-specific and all-cause mortality is increased by 2-2.5 times in those patients who are depressed compared to patients who are not depressed ${ }^{1}$.

\section{Moderator analyses by tumor stage}

Moderator analyses testing whether age moderates effects of depression on mortality only demonstrated a significant interaction effect of depression and age on all-cause mortality in patients with stage I breast cancer ${ }^{1}$ (Table 3). Younger patients at the age of 45 (1 SD below the mean) with depression had an almost tenfold increase in hazard of mortality compared to non-depressed patients $(\mathrm{HR}=9.82$ (95\% CI 2.26-42.68), $p=0.002$ ). Breast cancer patients

\footnotetext{
${ }^{1}$ Due to the fact that in patients with stage I breast cancer only one depressed patient died of the disease, it was not possible to calculate breast cancer-specific mortality in this subgroup.
} 
Table 1 Patient characteristics

\begin{tabular}{|c|c|c|c|}
\hline & $\begin{array}{l}\text { Stage I-III } \\
(n=1,604)\end{array}$ & $\begin{array}{l}\text { Stage IV } \\
(n=42)\end{array}$ & All $(n=1,646)$ \\
\hline $\begin{array}{l}\text { Age at } \\
\text { diagnosis } \\
\text { (mean } \\
(\mathrm{SD}))\end{array}$ & $56.13(12.57)$ & $50.36(24.14)$ & $56.19(12.54)$ \\
\hline \multicolumn{4}{|c|}{ Living situation } \\
\hline Not alone & $1,233(76.9 \%)$ & $28(66.7 \%)$ & $1,261(77.3 \%)$ \\
\hline Alone & $358(22.3 \%)$ & $12(28.6 \%)$ & $370(22.5 \%)$ \\
\hline Unknown & $13(0.8 \%)$ & $2(4.8 \%)$ & $15(0.9 \%)$ \\
\hline \multicolumn{4}{|l|}{ Stage } \\
\hline I & $804(50.1 \%)$ & & $804(48.8 \%)$ \\
\hline II & $580(36.2 \%)$ & & $580(35.2 \%)$ \\
\hline III & $220(13.7 \%)$ & & $220(13.4 \%)$ \\
\hline IV & & $42(100.0 \%)$ & $42(2.6 \%)$ \\
\hline \multicolumn{4}{|l|}{ Grade } \\
\hline 1 & $366(22.8 \%)$ & $4(9.5 \%)$ & $370(22.5 \%)$ \\
\hline 2 & $663(41.3 \%)$ & $15(35.7 \%)$ & $678(41.2 \%)$ \\
\hline 3 & $575(35.8 \%)$ & $23(54.8 \%)$ & $598(36.3 \%)$ \\
\hline \multicolumn{4}{|l|}{ Histology } \\
\hline $\begin{array}{l}\text { Invasive } \\
\text { ductal }\end{array}$ & $1,339(83.5 \%)$ & $34(81.0 \%)$ & $1,339(83.5 \%)$ \\
\hline Lobular & $145(9.0 \%)$ & $4(9.5 \%)$ & $145(9.0 \%)$ \\
\hline Other & $120(7.5 \%)$ & $4(9.5 \%)$ & $120(7.5 \%)$ \\
\hline \multicolumn{4}{|c|}{ Estrogen receptor } \\
\hline 0 & $265(16.5 \%)$ & $6(14.3 \%)$ & $267(16.2 \%)$ \\
\hline 1 & $57(3.6 \%)$ & $0(0.0 \%)$ & $57(3.5 \%)$ \\
\hline 2 & $202(12.6 \%)$ & $5(11.9 \%)$ & $207(12.6 \%)$ \\
\hline 3 & $1,079(67.3 \%)$ & $31(73.8 \%)$ & $1,110(67.4 \%)$ \\
\hline Unknown & $1(0.1 \%)$ & $0(0.0 \%)$ & $5(0.3 \%)$ \\
\hline \multicolumn{4}{|c|}{ Progesterone receptor } \\
\hline 0 & $403(25.1 \%)$ & $7(16.7 \%)$ & $410(24.9 \%)$ \\
\hline 1 & $149(9.3 \%)$ & $5(11.9 \%)$ & $154(9.4 \%)$ \\
\hline 2 & $325(20.3 \%)$ & $8(19.0 \%)$ & $333(20.2 \%)$ \\
\hline 3 & $505(31.5 \%)$ & $14(33.3 \%)$ & $519(31.5 \%)$ \\
\hline Unknown & $222(13.8 \%)$ & $8(19.0 \%)$ & $230(14.0 \%)$ \\
\hline \multicolumn{4}{|c|}{ Her2-neu status } \\
\hline 0 & $1,069(66.6 \%)$ & $26(61.9 \%)$ & $1,095(66.5 \%)$ \\
\hline 1 & $170(10.6 \%)$ & $4(9.5 \%)$ & $174(10.6 \%)$ \\
\hline 2 & $174(10.8 \%)$ & $7(16.7 \%)$ & $181(11.0 \%)$ \\
\hline 3 & $191(11.9 \%)$ & $5(11.9 \%)$ & $196(11.9 \%)$ \\
\hline \multicolumn{4}{|l|}{ Surgery } \\
\hline Yes & $1,594(99.4 \%)$ & $27(64.3 \%)$ & $1,609(97.8 \%)$ \\
\hline No & $10(0.6 \%)$ & $15(35.7 \%)$ & $37(2.2 \%)$ \\
\hline \multicolumn{4}{|l|}{ Radiation } \\
\hline Yes & $1,212(75.6 \%)$ & $12(28.6 \%)$ & $1,242(75.5 \%)$ \\
\hline No & $392(24.4 \%)$ & $30(71.4 \%)$ & $404(24.5 \%)$ \\
\hline \multicolumn{4}{|c|}{ Chemotherapy } \\
\hline Yes & $761(47.4 \%)$ & $21(50.0 \%)$ & $782(47.5 \%)$ \\
\hline No & $834(52.6 \%)$ & $21(50.0 \%)$ & $864(52.5 \%)$ \\
\hline
\end{tabular}

Table 1 continued

\begin{tabular}{|c|c|c|c|}
\hline & $\begin{array}{l}\text { Stage I-III } \\
(n=1,604)\end{array}$ & $\begin{array}{l}\text { Stage IV } \\
(n=42)\end{array}$ & All $(n=1,646)$ \\
\hline \multicolumn{4}{|c|}{ Hormonal therapy } \\
\hline Yes & $1,182(73.7 \%)$ & $9(21.4 \%)$ & $1,215(73.8 \%)$ \\
\hline No & $422(26.3 \%)$ & $33(78.6 \%)$ & $431(26.2 \%)$ \\
\hline \multicolumn{4}{|l|}{ Depression } \\
\hline Depressed & $250(15.6 \%)$ & $10(23.8 \%)$ & $260(15.8 \%)$ \\
\hline $\begin{array}{l}\text { Not } \\
\text { depressed }\end{array}$ & $1,354(84.4 \%)$ & $32(76.2 \%)$ & $1,386(84.2 \%)$ \\
\hline \multicolumn{4}{|c|}{ All-cause mortality } \\
\hline All & $176(11.0 \%)$ & $28(66.7 \%)$ & $204(12.4 \%)$ \\
\hline Depressed & $38(15.2 \%)$ & $7(70 \%)$ & $45(17.3 \%)$ \\
\hline $\begin{array}{l}\text { Not } \\
\text { depressed }\end{array}$ & $138(10.2 \%)$ & $21(65.6 \%)$ & $159(11.5 \%)$ \\
\hline \multicolumn{4}{|c|}{ Breast cancer mortality } \\
\hline All & $102(6.4 \%)$ & $24(57.1 \%)$ & $126(7.7 \%)$ \\
\hline Depressed & $26(10.4 \%)$ & $5(50.0 \%)$ & $31(11.9 \%)$ \\
\hline $\begin{array}{l}\text { Not } \\
\text { depressed }\end{array}$ & $76(5.6 \%)$ & $19(59.4 \%)$ & $95(6.9 \%)$ \\
\hline $\begin{array}{l}\text { Median } \\
\text { length of } \\
\text { survival } \\
\text { (months) }\end{array}$ & 74 & 49 & 74 \\
\hline $\begin{array}{l}\text { Median } \\
\text { length of } \\
\text { follow-up } \\
\text { (months) }\end{array}$ & 76 & 76.5 & 76 \\
\hline \multicolumn{4}{|c|}{ Total person time (months) } \\
\hline All & 118,162 & 2,115 & 120,277 \\
\hline Depressed & 18,406 & 527 & 18,933 \\
\hline $\begin{array}{l}\text { Not } \\
\text { depressed }\end{array}$ & 99,756 & 1,588 & 101,344 \\
\hline
\end{tabular}

with a mean age of 57 had a 3.7-fold increase in hazard of mortality if they were depressed (HR $=3.69(95 \% \mathrm{CI}$ 1.44-9.48), $p=0.007$ ). Older patients at age 69 (1 SD above the mean) who were depressed showed a $42 \%$ increase in hazard of mortality compared to non-depressed older patients with breast cancer. However, this effect was not significant $\quad(\mathrm{HR}=1.42 \quad(95 \%$ CI $0.48-4.18)$, $p=0.530$ ) (Fig. 2).

In neither of the other stages (II-IV) did age moderate effects of depression on breast cancer-specific or all-cause mortality (Table 3 ).

\section{Discussion}

The present study prospectively examined the role of depression at the time of diagnosis in predicting mortality in patients with breast cancer. In patients with curable breast cancer (i.e., stages I-III), results demonstrated a 
Table 2 Cox proportional-hazards regression analyses of patients with curable cancer $(n=1,604)$

\begin{tabular}{|c|c|c|c|c|c|}
\hline & & \multicolumn{2}{|c|}{ Breast cancer mortality } & \multicolumn{2}{|l|}{ All-cause mortality } \\
\hline & & HR $(95 \%$ CI $)$ & $p$ value & $\mathrm{HR}(95 \% \mathrm{CI})$ & $p$ value \\
\hline 1 & Age & $0.99(0.98-1.01)$ & 0.404 & $1.03(1.02-1.04)$ & $<0.001$ \\
\hline \multirow[t]{11}{*}{2} & Age & $1.00(0.99-1.02)$ & 0.651 & $1.04(1.02-1.05)$ & $<0.001$ \\
\hline & Stage & & & & \\
\hline & I & 1 & & 1 & \\
\hline & II & $6.32(3.09-12.92)$ & $<0.001$ & $2.93(1.97-4.37)$ & $<0.001$ \\
\hline & III & $12.42(5.94-25.97)$ & $<0.001$ & $5.21(3.36-8.05)$ & $<0.001$ \\
\hline & Grade & $1.92(1.31-2.82)$ & 0.001 & $1.53(1.18-1.99)$ & 0.001 \\
\hline & Histology & & & & \\
\hline & Invasive ductal & 1 & & 1 & \\
\hline & Lobular & $1.15(0.53-2.49)$ & 0.720 & $1.10(0.65-1.86)$ & 0.729 \\
\hline & Other & $1.19(0.57-2.47)$ & 0.645 & $1.12(0.65-1.95)$ & 0.684 \\
\hline & Estrogen receptor & $0.83(0.71-0.98)$ & 0.023 & $0.90(0.80-1.03)$ & 0.115 \\
\hline & Her2-neu status & $0.99(0.84-1.17)$ & 0.916 & $1.01(0.88-1.15)$ & 0.938 \\
\hline \multirow[t]{22}{*}{3} & Age & $0.99(0.98-1.01)$ & 0.532 & $1.03(1.01-1.04)$ & $<0.001$ \\
\hline & Stage & & & & \\
\hline & I & & & 1 & \\
\hline & II & $8.09(3.88-16.87)$ & $<0.001$ & $3.61(2.39-5.46)$ & $<0.001$ \\
\hline & III & $17.72(8.09-38.85)$ & $<0.001$ & $7.28(4.50-11.75)$ & $<0.001$ \\
\hline & Grade & $2.36(1.58-3.54)$ & $<0.001$ & $1.73(1.32-2.26)$ & $<0.001$ \\
\hline & Histology & & & & \\
\hline & Invasive ductal & 1 & & 1 & \\
\hline & Lobular & $1.20(0.55-2.61)$ & 0.676 & $1.08(0.64-1.82)$ & 0.782 \\
\hline & Other & $0.84(0.38-1.88)$ & 0.678 & $0.91(0.50-1.65)$ & 0.752 \\
\hline & Estrogen receptor & $0.87(0.70-1.10)$ & 0.200 & $0.84(0.70-1.01)$ & 0.063 \\
\hline & Her2-neu status & $1.03(0.87-1.2)$ & 0.751 & $1.04(0.91-1.20)$ & 0.532 \\
\hline & Surgery $^{\mathrm{a}}$ & 1 & & 1 & \\
\hline & & $0.09(0.03-0.30)$ & $<0.001$ & $0.02(0.00-0.09)$ & $<0.001$ \\
\hline & Radiation $^{\mathrm{a}}$ & 1 & & 1 & \\
\hline & & $1.88(1.04-3.33)$ & 0.028 & $1.10(0.76-1.58)$ & 0.631 \\
\hline & Chemotherapy $^{\mathrm{a}}$ & 1 & & 1 & \\
\hline & & $0.35(0.20-0.60)$ & $<0.001$ & $0.44(0.29-0.67)$ & $<0.001$ \\
\hline & Hormonal therapy $^{\mathrm{a}}$ & 1 & & 1 & \\
\hline & & $0.05(0.01-0.17)$ & $<0.001$ & $0.11(0.04-0.28)$ & $<0.001$ \\
\hline & Surgery $\times$ time & & & $1.07(1.01-1.14)$ & 0.025 \\
\hline & Hormonal therapy $\times$ time & $1.06(1.03-1.09)$ & $<0.001$ & $1.05(1.03-1.07)$ & $<0.001$ \\
\hline \multirow[t]{10}{*}{4} & Age & $1.00(0.98-1.02)$ & 0.667 & $1.03(1.01-1.04)$ & $<0.001$ \\
\hline & Stage & & & & \\
\hline & I & 1 & & 1 & \\
\hline & II & $8.12(3.90-16.92)$ & $<0.001$ & $3.61(2.39-5.46)$ & $<0.001$ \\
\hline & III & $16.90(7.71-37.06)$ & $<0.001$ & $6.94(4.30-11.21)$ & $<0.001$ \\
\hline & Grade & $2.37(1.58-3.56)$ & $<0.001$ & $1.74(1.33-2.27)$ & $<0.001$ \\
\hline & Histology & & & & \\
\hline & Invasive ductal & 1 & & 1 & \\
\hline & Lobular & $1.15(0.53-2.51)$ & 0.719 & $1.05(0.62-1.79)$ & 0.846 \\
\hline & Other & $0.84(0.37-1.90)$ & 0.676 & $0.91(0.50-1.66)$ & 0.761 \\
\hline
\end{tabular}


Table 2 continued

\begin{tabular}{|c|c|c|c|c|c|}
\hline & & \multicolumn{2}{|c|}{ Breast cancer mortality } & \multicolumn{2}{|l|}{ All-cause mortality } \\
\hline & & HR $(95 \%$ CI $)$ & $p$ value & HR (95 \% CI) & $p$ value \\
\hline & Estrogen receptor & $0.89(0.71-1.12)$ & 0.321 & $0.84(0.70-1.02)$ & 0.073 \\
\hline & Her2-neu status & $1.02(0.86-1.20)$ & 0.857 & $1.03(0.90-1.18)$ & 0.640 \\
\hline & Surgery $^{\mathrm{a}}$ & 1 & & 1 & \\
\hline & & $0.09(0.03-0.30)$ & $<0.001$ & $0.02(0.00-0.10)$ & $<0.001$ \\
\hline & Radiation $^{\mathrm{a}}$ & 1 & & 1 & \\
\hline & & $1.87(1.04-3.36)$ & 0.036 & $1.10(0.76-1.59)$ & 0.631 \\
\hline & Chemotherapy $^{\mathrm{a}}$ & 1 & & 1 & \\
\hline & & $0.36(0.21-0.62)$ & $<0.001$ & $0.46(0.30-0.70)$ & $<0.001$ \\
\hline & Hormonal therapy ${ }^{\mathrm{a}}$ & 1 & & 1 & \\
\hline & & $0.05(0.01-0.17)$ & $<0.001$ & $0.11(0.04-0.28)$ & $<0.001$ \\
\hline & Depression & & & & \\
\hline & Not depressed & 1 & & 1 & \\
\hline & Depressed & $1.51(0.95-2.41)$ & 0.084 & $1.54(1.06-2.25)$ & 0.024 \\
\hline & Surgery $\times$ time & & & $1.07(1.01-1.13)$ & 0.033 \\
\hline & Hormonal therapy $\times$ time & $1.06(1.03-1.09)$ & $<0.001$ & $1.05(1.03-1.07)$ & $<0.001$ \\
\hline \multirow[t]{26}{*}{5} & Age & $1.00(0.98-1.02)$ & 0.971 & $1.03(1.02-1.05)$ & $<0.001$ \\
\hline & Stage & & & & \\
\hline & I & 1 & & 1 & \\
\hline & II & $8.01(3.84-16.70)$ & $<0.001$ & $3.54(2.34-5.36)$ & $<0.001$ \\
\hline & III & $16.79(7.65-36.84)$ & $<0.001$ & $6.85(4.24-11.06)$ & $<0.001$ \\
\hline & Grade & $2.36(1.58-3.55)$ & $<0.001$ & $1.73(1.32-2.26)$ & $<0.001$ \\
\hline & Histology & & & & \\
\hline & Invasive ductal & 1 & & 1 & \\
\hline & Lobular & $1.16(0.53-2.52)$ & 0.713 & $1.05(0.62-1.78)$ & 0.851 \\
\hline & Other & $0.83(0.37-1.88)$ & 0.658 & $0.90(0.49-1.64)$ & 0.730 \\
\hline & Estrogen receptor & $0.90(0.71-1.13)$ & 0.349 & $0.85(0.71-1.02)$ & 0.084 \\
\hline & Her2-neu status & $1.01(0.85-1.19)$ & 0.916 & $1.03(0.90-1.18)$ & 0.687 \\
\hline & Surgery $^{\mathrm{a}}$ & 1 & & 1 & \\
\hline & & $0.09(0.03-0.30)$ & $<0.001$ & $0.02(0.00-0.10)$ & $<0.001$ \\
\hline & Radiation $^{\mathrm{a}}$ & 1 & & 1 & \\
\hline & & $1.87(1.04-3.36)$ & 0.035 & $1.10(0.76-1.60)$ & 0.605 \\
\hline & Chemotherapy $^{\mathrm{a}}$ & 1 & & 1 & \\
\hline & & $0.37(0.21-0.63)$ & $<0.001$ & $0.46(0.30-0.70)$ & $<0.001$ \\
\hline & Hormonal therapy ${ }^{\mathrm{a}}$ & 1 & & 1 & \\
\hline & & $0.05(0.01-0.16)$ & $<0.001$ & $0.11(0.04-0.28)$ & $<0.001$ \\
\hline & Depression & & & & \\
\hline & Not depressed & 1 & & 1 & \\
\hline & Depressed & $1.36(0.80-2.33)$ & 0.259 & $1.49(1.00-2.21)$ & 0.049 \\
\hline & Surgery $\times$ time & & & $1.07(1.01-1.13)$ & 0.034 \\
\hline & Hormonal therapy $\times$ time & $1.06(1.03-1.09)$ & $<0.001$ & $1.05(1.03-1.07)$ & $<0.001$ \\
\hline & Depression $\times$ age & $0.98(0.94-1.02)$ & 0.360 & $0.98(0.94-1.01)$ & 0.155 \\
\hline
\end{tabular}

Test statistics represent hazard ratios with $95 \%$ confidence interval (HR $(95 \% \mathrm{CI})$ )

${ }^{\text {a }}$ Referent signifies no treatment

$50 \%$ increase in hazard of mortality in depressed versus non-depressed patients. Analyses by tumor stage revealed that the effect of depression on mortality is for the most part driven by patients with earlier stage breast cancer (stages I and II) where the hazard of mortality for breast cancer-specific and all-cause mortality was 100-150\% 


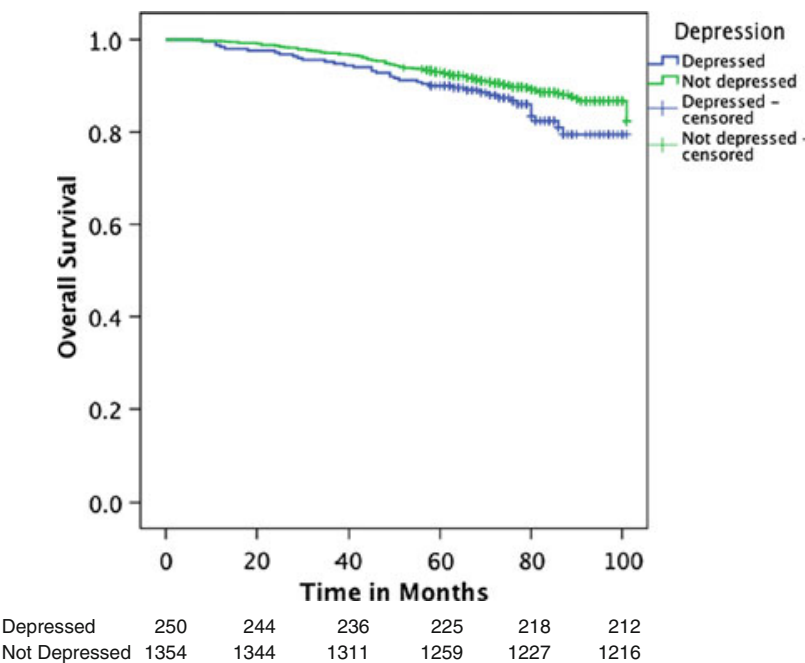

Fig. 1 Depression as a predictor of overall survival in patients with curable breast cancer (stages I-III)

increased in the depressed patients compared to nondepressed patients with breast cancer. No significant effect of depression on mortality in patients with breast cancer stages III and IV was found.

Furthermore, it was shown that age moderated this effect in patients with stage I breast cancer. In these patients, an age of 45 (1 SD below the mean) was associated with an almost tenfold increase in hazard of all-cause mortality if patients were depressed. This hazard decreased with age. While the hazard of mortality was still increased by $270 \%$ in those age 57 years (the mean age of the sample), a nonsignificant $40 \%$ increase in hazard of mortality was shown at age 69 (1 SD above the mean) in depressed older patients with early breast cancer.
As a consequence of advances in treatment for breast cancer, survival rates have increased over the last two decades, (as of 2007, 5-year survival rates for curable breast cancer were $89 \%$ [28]). Consequently, biologic variables may play less of a role in breast cancer than in other cancers with less favorable prognoses. The results of the present study support this assumption, since significant associations of depression on mortality could be demonstrated in breast cancer stages I and II, whereas no relationships could be shown for patients with stages III or IV breast cancer. In cancers with good prognoses, lifestyle factors may have a greater influence on outcome than in cancers with poor prognoses. Given that individuals with depression often present with an unhealthy lifestyle characterized by poor diet, alcohol abuse, smoking, and lack of physical activity [29, 30], these factors may play an important role in determining outcomes in cancer types with otherwise good prognoses.

However, effects of depression on mortality were largest in younger women with breast cancer, who on average have a poorer prognosis than older women with breast cancer. Due to its side effects, overall adherence rates to hormonal treatment are only moderate [31]. For younger premenopausal women, systemic and hormonal treatments are accompanied by a tremendous decrease in quality of life compared to postmenopausal women. Therefore, adherence to hormonal treatment might be lower among younger women with breast cancer [32] and may depend on the severity and the perceptions of treatment side effects. Hormonal treatment is more likely to threaten young women's self-concept of being young and fertile, and as a consequence also has an impact on their selfesteem [33-35]. Therefore, it is possible that patients high

Table 3 Cox proportional-hazards regression analyses of depression predicting mortality

\begin{tabular}{|c|c|c|c|c|}
\hline & \multicolumn{2}{|c|}{ Breast cancer mortality } & \multicolumn{2}{|c|}{ All-cause mortality } \\
\hline & $\mathrm{HR}(95 \% \mathrm{CI})$ & $p$ value & $\mathrm{HR}(95 \% \mathrm{CI})$ & $p$ value \\
\hline \multicolumn{5}{|l|}{ Stage I $(n=804)$} \\
\hline Depression & $\mathrm{n} / \mathrm{a}$ & $\mathrm{n} / \mathrm{a}$ & $2.30(1.02-5.15)$ & 0.044 \\
\hline Depression $\times$ age & $\mathrm{n} / \mathrm{a}$ & $\mathrm{n} / \mathrm{a}$ & $0.92(0.86-0.99)$ & 0.030 \\
\hline \multicolumn{5}{|l|}{ Stage II $(n=580)$} \\
\hline Depression & $2.53(1.32-4.86)$ & 0.005 & $2.01(1.16-3.48)$ & 0.013 \\
\hline Depression $\times$ age & $1.01(0.95-1.08)$ & 0.735 & $1.00(0.95-1.06)$ & 0.918 \\
\hline \multicolumn{5}{|l|}{ Stage III $(n=220)$} \\
\hline Depression & $0.99(0.47-2.10)$ & 0.979 & $0.92(0.47-1.80)$ & 0.801 \\
\hline Depression $\times$ age & $0.99(0.91-1.07)$ & 0.718 & $0.95(0.89-1.02)$ & 0.144 \\
\hline \multicolumn{5}{|l|}{ Stage IV $(n=42)$} \\
\hline Depression & $0.57(0.16-2.03)$ & 0.388 & $1.15(0.38-3.46)$ & 0.807 \\
\hline Depression $\times$ age & $0.95(0.78-1.16)$ & 0.606 & $1.06(0.93-1.21)$ & 0.403 \\
\hline
\end{tabular}

Separate analyses by tumor stage with control of the variables listed in Table 2. Proportionality of hazards was tested within each stage and for each outcome and adjusted accordingly. Test statistics represent hazard ratios with $95 \%$ confidence interval (HR (95\% CI)) 

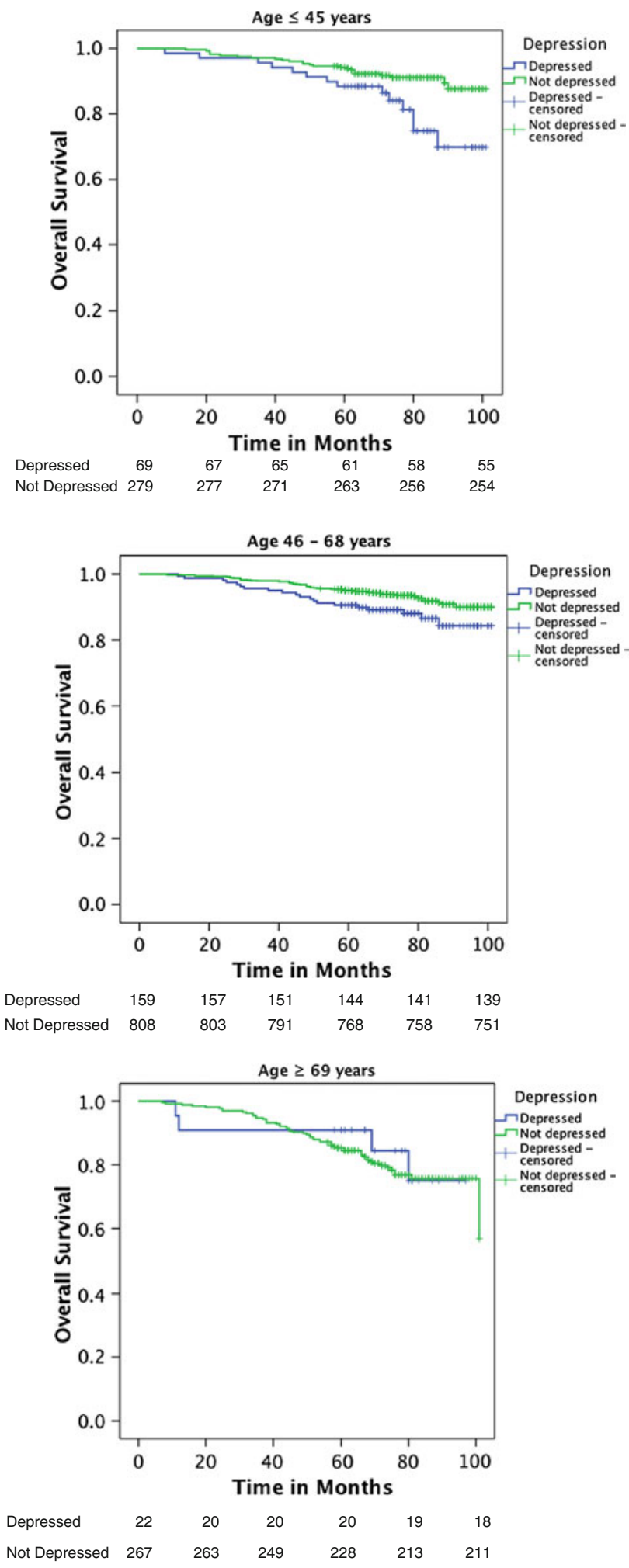

Fig. 2 Age moderating the relationship between depression and overall survival in stage I breast cancer. Age $\leq 45$ years are patients at least 1 SD below the mean, age 46-68 years are patients within 1 SD above or below the mean, and age $\geq 69$ years are patients 1 SD above the mean in depression may demonstrate poorer adherence to hormonal treatment as they likely do worse in dealing with its side effects [36]. Thus, adherence may represent an age dependent mediator that places younger depressed women with breast cancer at increased risk of mortality. Given that data on adherence to hormonal treatment were not available, we were unable to test this potential mechanism.

Beyond this more behavioral pathway, symptoms of depression at diagnosis may reveal a woman's emotional stress response that coincides with her biologic reactivity to stressors. This response is likely marked by endocrine and immunologic changes that may elevate a patient's biologic vulnerability to cancer progression [37, 38]. In particular, inflammation may play an important role in mediating effects of depression on mortality. Depression is also known to influence the stress hormone cortisol through abnormal HPA axis functioning. This process may represent a second mechanism which might explain poorer outcome in depressed patients with breast cancer. Again, younger women with breast cancer who are depressed may show greater biologic reactivity since they may have more difficulty coping with cancer and may be more distressed because of multiple roles and their demands (e.g., raising under-aged children, having a larger household, managing work or a career) which tend to decrease with age.

The predictive relationship of depression could be more consistently demonstrated for all-cause compared to breast cancer-specific mortality. It is well-known that the reliability of the documentation of breast cancer-specific survival is not as high as for overall survival, and is therefore less valid. However, there were few discrepancies in findings between all-cause and breast cancer-specific mortality; the missing significance of depression as a predictor of breast cancer-specific mortality approached significance and the hazard ratio was the same size as the hazard ratio for all-cause mortality. The majority of previous studies did not differentiate between breast cancerspecific and overall survival.

This study should be interpreted in the context of its strengths and limitations. The relatively brief depression measure does not substitute for a diagnosis of depression based on a structured clinical interview. However, this study was population-based in nature and thus a timeintensive and costly assessment of mental disorders via structured interviews was not feasible, thus necessitating the use of an established cancer-specific screening tool. Although the use of a structured clinical interview would have theoretically been superior, it most likely would have resulted in a significant increase in patients declining participation in a study with a much longer time commitment (which a structured clinical interview would have required) 
at the time of cancer diagnosis. Furthermore, this likely would result in a selection bias, where younger, fitter patients would be more likely to participate [39].

Conversely, some misclassifications may have occurred with the approach chosen. Responders and non-responders to the screening questionnaire did not differ in any clinical variables except for an average age difference of 2.6 years, and therefore, the slightly younger patient sample may have been more distressed than breast cancer patients in British Columbia on average. However, prevalence rates of depression in the current study match well with results from other larger scale studies on women with breast cancer [40, 41].

Further, the epidemiological nature of this study prevented us from collecting data on depression over time in order to consider various trajectories of depression and how these may be related to mortality. This is an important area for future research to examine. Further, although the sample was well described in terms of tumor stage, grade, hormone receptor status, and treatments received, no data had been collected for performance status, reproductive information, or comorbidities. Performance status in particular is a strong predictor of survival, but is correlated with mental health. As well, only few demographic variables had been documented.

The assessment of depression at a fixed time point, namely post-cancer diagnosis, reduces error variance. Consequently, assessments were not confounded with emotional reactions arising from cancer treatment and its side effects. However, because the depression measure was collected before patients were given their prognosis and treatment plan, there may have been reduced variance in depression scores. This may have served to make the results of this study conservative. As well, patients were recruited within a routine program for emotional distress screening. Hence, the sample can be considered representative of the Greater Vancouver region and of sufficient size such that analyses do not suffer a lack of statistical power.

\section{Directions for future research}

Whether effective treatment of depression improves survival in patients with breast cancer remains to be determined. Some evidence suggests that a decrease in depression in patients with metastatic breast cancer is associated with lower mortality [42]. Following the observational findings of this study, it would be interesting to explore whether there are benefits to survival when patients high in depression make use of the various sources of counseling or psychotherapy that have been established for women with breast cancer in the last few years. As well, exploring the two mechanisms (i.e. positive behavior change in life style and adherence, decrease in inflammation) proposed, explaining differences in survival among depressed as opposed to not depressed patients with breast cancer in intervention studies, would result in a better understanding of the associations identified in the present study.

\section{Clinical implications}

The great impact of depression on mortality, especially in younger women with early stage breast cancer, exemplifies the importance of routine screening programs for depression in the cancer care context. Along with systematic screening, there must be a consistent referral procedure in place so that depressed patients receive psychological treatment in a timely manner. A broad spectrum of techniques can support patients in overcoming their depression and coping with a cancer diagnosis. Cognitive-behavioral therapy has been shown to be the most efficacious method of treatment for depression, and not only treats symptoms of depression but can also influence behavioral factors that are related to cancer treatment and survivorship [43, 44]. Whether antidepressant treatment alone can replace cognitive-behavioral therapy in terms of its effects in reducing depressive symptoms and behaviors remains to be determined. It is likely that antidepressants represent a supportive treatment but might not be able to promote favorable behavior change.

Acknowledgments We are grateful to Colleen Wong, CHIM, from the BC Cancer Agency, Surveillance \& Outcomes Unit-Population Oncology, for provision of survival data. Supported by the Canadian Institute for Health Research (CIHR Team for Supportive Cancer Care; \#AQC83559). There are no financial interests associated with this work.

Ethical standards This study complies with Canadian ethical standards and was approved by the joint UBC \& BCCA research ethics board.

Open Access This article is distributed under the terms of the Creative Commons Attribution Noncommercial License which permits any noncommercial use, distribution, and reproduction in any medium, provided the original author(s) and the source are credited.

\section{References}

1. Derogatis LR, Abeloff MD, Melisaratos N (1979) Psychological coping mechanisms and survival time in metastatic breast cancer. JAMA 242:1504-1508

2. Bardwell WA, Natarajan L, Dimsdale JE, Rock CL, Mortimer JE, Hollenbach K, Pierce JP (2006) Objective cancer-related variables are not associated with depressive symptoms in women treated for early-stage breast cancer. J Clin Oncol 24:2420-2427

3. van't Spijker A, Trijsburg RW, Duivenvoorden HJ (1997) Psychological sequelae of cancer diagnosis: a meta-analytical review of 58 studies after 1980. Psychosom Med 59:280-293

4. Satin JR, Linden W, Phillips MJ (2009) Depression as a predictor of disease progression and mortality in cancer patients. Cancer 115:5349-5361 
5. Pinquart M, Duberstein PR (2010) Depression and cancer mortality: a meta-analysis. Psychol Med 40:1797-1810

6. Hislop TG, Waxler NE, Coldman AJ, Elwood JM, Kan L (1987) The prognostic significance of psychosocial factors in women with breast cancer. J Chronic Dis 40:729-735

7. Tross S, Herndon J 2nd, Korzun A, Kornblith AB, Cella DF, Holland JF, Raich P, Johnson A, Kiang DT, Perloff M, Norton L, Wood W, Holland JC (1996) Psychological symptoms and diseasefree and overall survival in women with stage II breast cancer. Cancer and Leukemia Group B. J Natl Cancer Inst 88:661-667

8. Goodwin PJ, Ennis M, Bordeleau LJ, Pritchard KI, Trudeau ME, Koo J, Hood N (2004) Health-related quality of life and psychosocial status in breast cancer prognosis: analysis of multiple variables. J Clin Oncol 22:4184-4192

9. Watson M, Homewood J, Haviland J, Bliss JM (2005) Influence of psychological response on breast cancer survival: 10-year followup of a population-based cohort. Eur J Cancer 41:1710-1714

10. Phillips KA, Osborne RH, Giles GG, Dite GS, Apicella C, Hopper JL, Milne RL (2008) Psychosocial factors and survival of young women with breast cancer: a population-based prospective cohort study. J Clin Oncol 26:4666-4671

11. Saquib N, Pierce JP, Saquib J, Flatt SW, Natarajan L, Bardwell WA, Patterson RE, Stefanick ML, Thomson CA, Rock CL, Jones LA, Gold EB, Karanja N, Parker BA (2011) Poor physical health predicts time to additional breast cancer events and mortality in breast cancer survivors. Psychooncology 20:252-259

12. Watson M, Haviland JS, Greer S, Davidson J, Bliss JM (1999) Influence of psychological response on survival in breast cancer: a population-based cohort study. Lancet 16:1331-1336

13. Onitilo AA, Nietert PJ, Egede LE (2006) Effect of depression on all-cause mortality in adults with cancer and differential effects by cancer site. Gen Hosp Psychiatry 28:396-402

14. Vodermaier A, Linden W, Siu C (2009) Screening for emotional distress in cancer care. A systematic review of assessment instruments. J Natl Cancer Inst 101:1464-1488

15. Zigmond AS, Snaith RP (1983) The hospital anxiety and depression scale. Acta Psychiatr Scand 67:361-370

16. Radloff LS (1977) The CES-D Scale: a self-report depression scale for research in the general population. Appl Psychol Meas $1: 385-401$

17. Hjerl K, Andersen EW, Keiding N, Mouridsenm HT, Mortensen PB, Jørgensen T (2003) Depression as a prognostic factor for breast cancer mortality. Psychosomatics 44:24-30

18. Goodwin JS, Zhang DD, Ostir GV (2004) Effect of depression on diagnosis, treatment, and survival of older women with breast cancer. J Am Geriatr Soc 52:106-111

19. Cao J, Olson R, Tyldesley S (Accepted 2013) Comparison of recurrence and survival rates between breast-conserving therapy and mastectomy in young women with breast cancer. Curr Oncol 20. doi: $10.3747 / \mathrm{co} .20 .1543$

20. de Bock GH, van der Hage JA, Putter H, Bonnema J, Bartelink H, van de Velde CJ (2006) Isolated loco-regional recurrence of breast cancer is more common in young patients and following breast conserving therapy: long-term results of European Organisation for Research and Treatment of Cancer studies. Eur J Cancer 42:351-356

21. Nixon AJ, Neuberg D, Hayes DF, Gelman R, Connolly JL, Schnitt $S$ et al (1994) Relationship of patient age to pathologic features of the tumor and prognosis for patients with stage I or II breast cancer. J Clin Oncol 12:888-894

22. Henderson AS, Jorm AF, Korten AE, Jacomb P, Christensen H, Rodgers B (1998) Symptoms of depression and anxiety during adult life: evidence for a decline in prevalence with age. Psychol Med 28:1321-1328

23. Vodermaier A, Linden W, MacKenzie R, Greig D, Marshall C (2011) Disease stage predicts post-diagnosis anxiety and depression only in some types of cancer. Br J Cancer 12:1814-1817
24. Linden W, Vodermaier A, McKenzie R, Greig D (2012) Anxiety and depression after cancer diagnosis: prevalence rates by cancer type, gender, and age. J Affect Disord 141:343-351

25. Linden W, Yi D, Barroetavena MC, MacKenzie R, Doll R (2005) Development and validation of a psychosocial screening instrument for cancer. Health Qual Life Outcomes 3:54

26. Linden W, Vodermaier A, MacKenzie R, Barroetavena M, Yi D, Doll R (2009) The Psychosocial Screen for Cancer (PSSCAN): further validation and normative data. Health Qual Life Outcomes $7: 16$

27. Bewick V, Cheek L, Ball J (2004) Statistics review 12: survival analysis. Crit Care 8:389-394

28. The surveillance and outcomes unit population oncology (2010) BC Cancer Agency Survival Statistics 2007. http://www.bccan cer.bc.ca/NR/rdonlyres/D8291ED8-FAEE-4692-A256-5320928 187F2/44155/Survival_Statistics_2007_Rerun.pdf. Accessed 25 May 2012

29. Lopresti AL, Hood SD, Drummond PD (2013) A review of lifestyle factors that contribute to important pathways associated with major depression: diet, sleep and exercise. J Affect Disord 148:12-27

30. Berk M, Sarris J, Coulson CE, Jacka FN (2013) Lifestyle management of unipolar depression. Acta Psychiatr Scand Suppl 443:38-54

31. Murphy CC, Bartholomew LK, Carpentier MY, Bluethmann SM, Vernon SW (2012) Adherence to adjuvant hormonal therapy among breast cancer survivors in clinical practice: a systematic review. Breast Cancer Res Treat 134:459-478

32. Güth U, Myrick ME, Kilic N, Eppenberger-Castori S, Schmid SM (2012) Compliance and persistence of endocrine adjuvant breast cancer therapy. Breast Cancer Res Treat 131:491-499

33. Avis NE, Crawford S, Manuel J (2005) Quality of life among younger women with breast cancer. J Clin Oncol 23:3322-3330

34. Schover LR (1994) Sexuality and body image in younger women with breast cancer. J Natl Cancer Inst Monogr 16:177-182

35. Wong-Kim EC, Bloom JR (2005) Depression experienced by young women newly diagnosed with breast cancer. Psychooncology 7:564-573

36. Cluze C, Rey D, Huiart L, BenDiane MK, Bouhnik AD, Berenger C, Carrieri MP, Giorgi R (2012) Adjuvant endocrine therapy with tamoxifen in young women with breast cancer: determinants of interruptions vary over time. Ann Oncol 23:882-890

37. Armaiz-Pena GN, Lutgendorf SK, Cole SW, Sood AK (2009) Neuroendocrine modulation of cancer progression. Brain Behav Immun 23:10-15

38. McEwen BS, Seeman T (1999) Protective and damaging effects of mediators of stress: elaborating and testing the concepts of allostasis and allostatic load. Ann NY Acad Sci 896:30-47

39. Olson R, Parkinson M, McKenzie M (2010) Selection bias introduced by neuropsychological assessments. Can J Neurol Sci 37:264-268

40. Hinz A, Krauss O, Hauss JP, Höckel M, Kortmann RD, Stolzenburg JU, Schwarz R (2010) Anxiety and depression in cancer patients compared with the general population. Eur J Cancer Care (Engl) 19:522-529

41. Hopwood P, Sumo G, Mills J, Haviland J, Bliss JM (2010) The course of anxiety and depression over 5 years of follow-up and risk factors in women with early breast cancer: results from the UK Standardisation of Radiotherapy Trials (START). Breast 19:84-91

42. Giese-Davis J, Collie K, Rancourt KM, Neri E, Kraemer HC, Spiegel D (2011) Decrease in depression symptoms is associated with longer survival in patients with metastatic breast cancer: a secondary analysis. J Clin Oncol 29:413-420

43. Duijts SF, Faber MM, Oldenburg HS, van Beurden M, Aaronson NK (2011) Effectiveness of behavioral techniques and physical exercise on psychosocial functioning and health-related quality of 
life in breast cancer patients and survivors-a meta-analysis. Psychooncology 20:115-126

44. Jacobsen PB, Phillips KM, Jim HS, Small BJ, Faul LA, Meade CD, Thompson L, Williams CC Jr, Loftus LS, Fishman M,
Wilson RW (2013) Effects of self-directed stress management training and home-based exercise on quality of life in cancer patients receiving chemotherapy: a randomized controlled trial. Psychooncology 22:1229-1235 